\title{
Commentary
}

\section{Rational approach to electronic fetal monitoring during labour in 'all' resource settings}

\author{
Edwin Chandraharan
}

Sri Lanka Journal of Obstetrics and Gynaecology 2010; 32: 77-84

\begin{abstract}
Electronic fetal monitoring (EFM) involves the use of cardiotocograph (CTG) to asses fetal wellbeing during labour in order to detect intrapartum fetal hypoxia early so as to institute timely and appropriate action. Indeed, when CTG was introduced into obstetric practice in the 1960s, it was expected to prevent perinatal brain injury and reduce the incidence of short term (neonatal admissions, seizures and neonatal deaths) and long term (cerebral palsy, learning difficulties) that arise secondary to intrapartum hypoxic insults. Unfortunately, although the rates of caesarean sections have dramatically increased over the last 40 years, there has been virtually no change in cerebral palsy rates. However, increasing caesarean section rates have contributed to maternal morbidity and mortality, including rising incidence of morbidly adherent placentae (placenta accrete, increta and percreta).
\end{abstract}

Apart from a high 'false positive' rate of CTG, failure to appreciate the pathophysiology of fetal heart rate changes by the clinicians have contributed to increased operative deliveries, without any substantial improvements in neonatal outcomes. Hence, a rational approach is warranted in the use and interpretation of CTGs to improve maternal and fetal outcomes, in all settings, irrespective of the availability of additional tests of fetal wellbeing such as fetal blood sampling (FBS), fetal electrocardiograph (ST analyser or STAN), fetal pulse oximetry or scalp lactate.

\section{Introduction}

Cardiotocograph (CTG) is a screening tool that is used to detect fetal hypoxia during labour. Fetal heart rate (FHR) is picked up by a transducer placed on the

\footnotetext{
${ }^{1}$ Lead Consultant Labour Ward and Lead for Clinical Governance in Obstetrics and Gynaecology, St. George's Healthcare NHS Trust, Blackshaw Road, London SW 17 ORE.
}

E-mail: edchandi@yahoo.co.uk maternal abdomen (or by an electrode attached to the fetal scalp) and this recorded on a paper (CTG 'trace'), similar to an adult electrocardiograph (ECG). This forms the 'cardiac' part of the fetal cardiotocograph. Another electrode is placed on the maternal abdomen to record the frequency and duration of the uterine contractions and this forms the 'toco' part of the fetal CTG. The latter is as important as the former and increased frequency and duration of uterine contractions are likely to affect uterine blood flow, leading to changes in the fetal heart rate.

It is the responsibility of clinicians managing women in labour to understand the normal physiology of fetal heart rate changes during labour, any deviation from this 'normal pattern' and the possible causes for such deviations. Overall management should take into consideration the type of hypoxia, the rapidity of development of hypoxia, the capacity of the fetus to respond to the given hypoxic insult as well as other clinical factors such as the rate of progress of labour, intrapartum risk factors (bleeding, meconium, maternal pyrexia or suspected chorioamnionitis) as well as presence of any correctable factors (uterine hyperstimulation, maternal dehydration or pyrexia). Failure to do so may result in an unnecessary emergency intervention during labour that may jeopardize the health and wellbeing of the mother, her baby or both. Conversely, failure to recognize abnormal features of the CTG trace which is commonly but incorrectly, termed as 'fetal distress' secondary to intrapartum hypoxia may result in metabolic acidosis. This can result in perinatal brain injury leading to hypoxic ischaemic encephalopathy (HIE), neonatal death or long term neurological sequelae $^{1}$. The 4 th confidential enquiries into stillbirths and deaths in infancy (CESDI) report in the United Kingdom concluded that sub-optimal care involving CTG interpretation and failure to act when a CTG abnormality was detected contributed to over half of all intrapartum related deaths ${ }^{2}$. Hence, it is essential to understand the CTG patterns and the underlying mechanisms that result in this pattern to optimize our management ${ }^{3}$.

Understanding the pathophysiology of fetal heart rate changes during labour

At the outset, it is important to recognize that 
human labour is a very stressful period for the fetus: its environment dramatically changes within a short period of time. The uterine walls that have been quiescent to allow the growth of the fetus during pregnancy, suddenly lose their 'passive' state. Instead, it contracts strongly and 'squeeze' the baby approximately 3-4 times every ten minutes, each contraction lasting for 40-60 seconds. The umbilical cord and head are likely to get compressed during contractions and many fetuses demonstrate an 'alarm' reaction by releasing catecholamines from the adrenal glands to cope with this stress, just like adults facing a stressful situation. Their heart rate is likely to show changes as a result of these mechanical stresses and will be recorded in the CTG trace. However, the fetus does not require any intervention as it is a physiological response to stress. Figure 1 illustrates various factors that affect the fetal heart rate during labour.

The ability of the fetus to mount a successful 'alarm' reaction to cope with the hypoxic or mechanical stress during labour would depend on the physiological reserve of the fetus (i.e. preterm, posterm or fetuses with intra-uterine growth restriction may have reduced utero-placental reserve as well as its inherent physiological mechanisms), rapidity of development of stress (i.e. time available for the fetus to defend itself through the release of catecholamines) and other factors such as presence of meconium, and infections (fetal infection decreases the ability to mount a successful response and also may potentiate the detrimental effects of hypoxia on the fetal brain).

\section{Factors that affect fetal heart rate during labour}

\section{Baro-receptors (Figure 1)}

Similar to adults, stimulation of the baro-receptors in the carotid sinus and aortic arch leads to activation of the parasympathetic nervous system that results in a fall in the fetal heart rate. This will be noted as a 'deceleration' on the CTG. Such a deceleration is usually short lasting and rapidly returns to the baseline heart rate as it is a 'reflex' neurological mechanism. Head compression and cord compressions are associated with activation of the parasympathetic nervous system and hence result in early and variable decelerations, both of these are short lasting. The fetus is not exposed to hypoxia during these decelerations (as they are due to mechanical compression) and hence, they do not warrant any action.

\section{Chemo-receptors (Figure 1)}

Peripheral chemoreceptors are located on the aortic and carotic bodies and the central chemoreceptors are situated within the brain. These respond to the changes in the chemical composition of the blood (i.e partial pressures of oxygen, carbon dioxide and the concentration of free hydrogen ions). Utero-placental insufficiency during labour results in accumulation of carbon dioxide and hydrogen ions due to metabolic acidosis that occurs secondary to anaerobic metabolism in the fetus. Increased carbon dioxide and hydrogen ion concentration coupled with decreased oxygen content of the fetal blood would

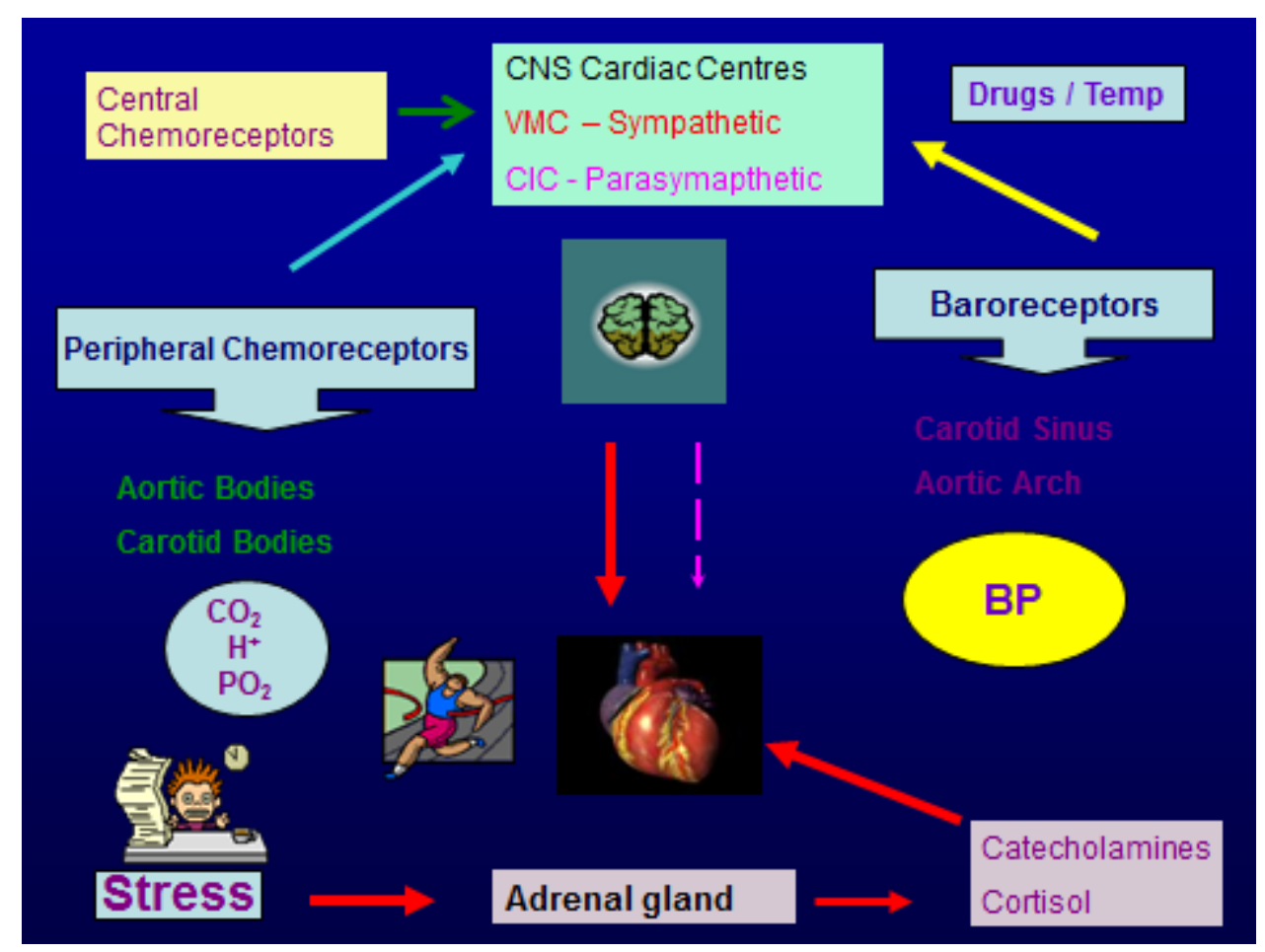

Figure 1 
stimulate the chemoreceptors. This results in parasympathetic activation leading to a fall in the fetal heart rate. However, unlike the baroreceptor mediated deceleration, the chemoreceptor mediated deceleration takes a longer time to recover to the normal baseline. This is because fresh maternal blood is required to 'wash-out' the accumulated acid and carbon dioxide, which takes time, leading to a 'lag time'. It is for this reason that 'late decelerations' noted on the CTG are considered ominous as they are likely to reflect a chemoreceptor mediated fall in the heart rate with a slow recovery to the baseline that occurs secondary to uteroplacental insufficiency. Hence, presence of metabolic acidosis is more likely.

\section{Role of the somatic nervous system}

Voluntary activities such as exercise increase the heart rate through the somatic nervous system. Similarly, heart rate increases due to fetal activity in utero, which is mediated through the fetal somatic nervous system and is recorded on the CTG as an 'acceleration'. These accelerations are considered as hallmarks of fetal wellbeing. A sick or hypoxic fetus exposed to significant intrapartum hypoxia would reduce its movements to conserve energy and therefore is unlikely to show accelerations on the CTG.

\section{Role of the fetal adrenal gland (Figure 1)}

Adrenal gland is responsible for the release of 'emergency hormones' such as cortisol and catecholamines (adrenaline and noradrenaline), all of which are essential for survival. Catecholamines have a sympathomimetic activity and hence increase the heart rate and force of contractions of the myocardium. When a fetus is exposed to continuing hypoxia over a time period, it relies on the adrenal gland to increase the heart rate to perfuse its essential organs (brain, heart and the adrenal gland, as all other functions are carried out by the palcenta) to survive in-utero.

\section{Exogenous factors}

Clinicians should not disregard multitude of other factors that may affect the fetal heart rate during labour. These include maternal temperature, infections, medications (betasympathomimetics, opioids, cardiac drugs administered to the mother), mechanical stimulation (vaginal examinations), maternal hypovolumia (epidural analgesia), maternal dehydration as well as fetal cardiac malformations and conductive defects.

\section{What is a 'Normal CTG'?}

Four features are considered when assessing fetal wellbeing in labour using a $\mathrm{CTG}^{4}$. These include the baseline fetal heart rate, baseline variability, accelerations and the presence of decelerations. Each of these features are further classified into 'reassuring, non-reassuring and abnormal' according to National Institute of Health and Clinical Excellence (NICE) Classification $^{5}$. Tables 1 and 2 illustrate feature classification as well as the overall classification as recommended by NICE. The use of such classification systems will help reduce variations in clinical practice and confusion due to different terminologies and may help improve outcomes.

For a CTG to be classified as 'normal' all the four parameters of the fetal heart trace should be within the 'reassuring category' (Tables 1 and 2). It is often easier to recognize a normal CTG as shown in Figure 2 and it has been shown that the risk of fetal hypoxia and acidosis is extremely small when the CTG is normal $^{6}$. False negatives may sometimes occur (poor outcome despite of a normal CTG). In such cases, factors other than hypoxia such as intrauterine infection, maternal pyrexia and fetal congenital or metabolic problems that may have contributed to poor fetal outcome should be explored. It is vital to consider the changes in baseline heart rate, variability and the presence of accelerations and decelerations over time (i.e. examine the entire CTG trace) as opposed to taking a 'snapshot view' of the CTG.

Figure 2 demonstrates the presence of accelerations ( $\geq 2$ episodes in 20 minutes, each rising at least 15 beats per minute above the baseline rate lasting for $\geq 15$ seconds) which are usually associated with fetal movements and appear to suggest an intact somatic nervous system. Similarly, the normal baseline variability indicates an intact autonomic nervous system. Hence, overall this demonstrates that both the somatic and the autonomic nervous systems are functioning well and therefore, the likelihood of intrapartum hypoxia is very small. Conversely, CTG features that were commonly associated with cerebral palsy were multiple late decelerations and decreased baseline variability ${ }^{7}$.

\section{How reliable is the CTG?}

It has been shown that when all the features of the CTG are reassuring and in the presence of accelerations, the likelihood of fetal hypoxia is very small ${ }^{6}$. Presence of cycling (alternating periods of quiescence and activity) as shown in Figure 3 is considered to be an excellent sign of fetal wellbeing as it appears to signify a neurologically intact fetus. Conversely, a prospective study of 6825 patients found the positive predictive value of an abnormal CTG trace for babies who needed intermittent positive pressure ventilation to be $8.7 \%{ }^{8}$. The same study reported a positive predictive value of an abnormal CTG trace to be $27.4 \%$ for all babies with an Apgar score of less 
than 7 at five minutes. It is, therefore, very important to understand that false positive rates of pathological CTGs with all features being abnormal were extremely high, with a low predictive value for cerebral palsy ${ }^{7}$. It is essential to incorporate antepartum and intrapartum risk factors and consider the entire clinical picture, whilst making a management plan for the fetus in question ${ }^{9}$. Therefore, CTG has a very high sensitivity for fetal hypoxia but has a poor specificity. Hence, if it is used alone, it is likely to increase the operative delivery rates and interventions without significantly improving perinatal outcome.

\section{Interpreting CTG using the knowledge of pathophysiology of intrapartum hypoxia}

Several additional tests of fetal wellbeing such as fetal blood sampling (FBS), fetal pulse oximetry, fetal electrocardiograph (ST-Segment Analyser or
STAN) and fetal scalp lactate have been described to reduce the false positive rate of $\mathrm{CTG}^{9,10,11}$. However, these additional tests are not universally available, especially in developing countries and this is likely to increase caesarean section rates, which may increase maternal morbidity and mortality without any demonstrable benefit to the fetus. Even in developed countries where these tests are universally available, the operative delivery rates are increasing without any significant improvement in cerebral palsy or perinatal mortality ${ }^{12}$. It is therefore pertinent that clinicians need to understand various types of hypoxia and their potential causes during labour to improve perinatal outcome as well as to reduce interventions.

A fetus who is admitted in labour with a normal CTG as shown in Figure 2 can be exposed to three types of hypoxia during labour: acute, sub-acute and gradually evolving.

Table 1. Categorizing the individual features of the CTG (NICE)

\begin{tabular}{|c|c|c|c|c|}
\hline Feature & Baseline (bpm) & Variability (bpm) & Decelerations & Accelerations \\
\hline Reassuring & $110-160$ & $\geq 5$ & None & Present \\
\hline Non-reassuring & $\begin{array}{l}100-109 \\
161-180\end{array}$ & $\begin{array}{l}<5 \text { for } \\
40-90 \text { minutes }\end{array}$ & $\begin{array}{l}\text { Typical variable } \\
\text { decelerations with } \\
\text { over } 50 \% \text { of contractions, } \\
\text { occurring for over } \\
90 \text { minutes } \\
\text { Single prolonged } \\
\text { deceleration for up to } \\
3 \text { minutes }\end{array}$ & $\begin{array}{l}\text { The absence of } \\
\text { accelerations with } \\
\text { otherwise normal } \\
\text { trace is of } \\
\text { uncertain } \\
\text { significance }\end{array}$ \\
\hline Abnormal & $\begin{array}{l}<100 \\
>180 \\
\text { Sinusoidal } \\
\text { pattern } \\
\geq 10 \text { minutes }\end{array}$ & $\begin{array}{l}<5 \text { for } \\
90 \text { minutes }\end{array}$ & $\begin{array}{l}\text { Either atypical variable } \\
\text { decelerations with over } \\
50 \% \text { of contractions or } \\
\text { late decelerations, both } \\
\text { for over } 30 \text { minutes } \\
\text { Single prolonged } \\
\text { deceleration for more } \\
\text { than } 3 \text { minutes }\end{array}$ & \\
\hline
\end{tabular}

Table 2 . Cardiotocograph (CTG) classification (NICE)

\begin{tabular}{ll}
\hline Category & Definition \\
\hline Normal & A CTG where all four features fall into the 'reassuring' category \\
\hline Suspicious & $\begin{array}{l}\text { A CTG where one of the features fall into 'non-reassuring category' } \\
\text { and the remainder of the features are reassuring }\end{array}$ \\
\hline Pathological & $\begin{array}{l}\text { A CTG whose features fall into two or more non-reassuring categories } \\
\text { or one or more abnormal categories }\end{array}$ \\
\hline
\end{tabular}




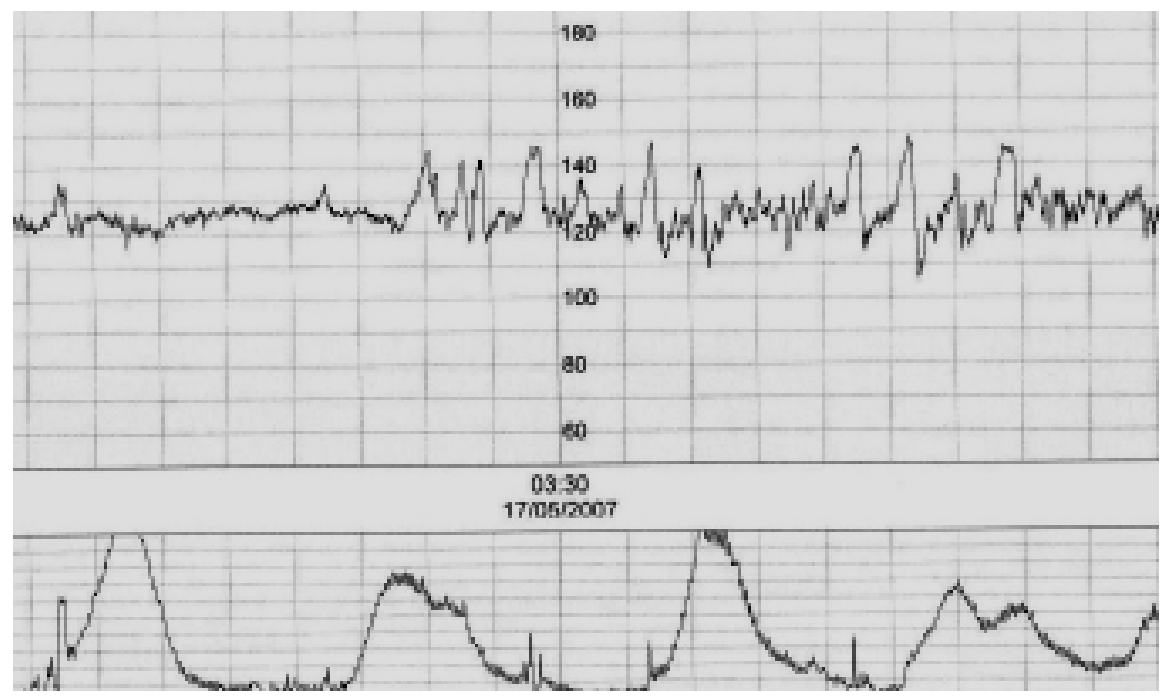

Figure 2. Normal CTG.

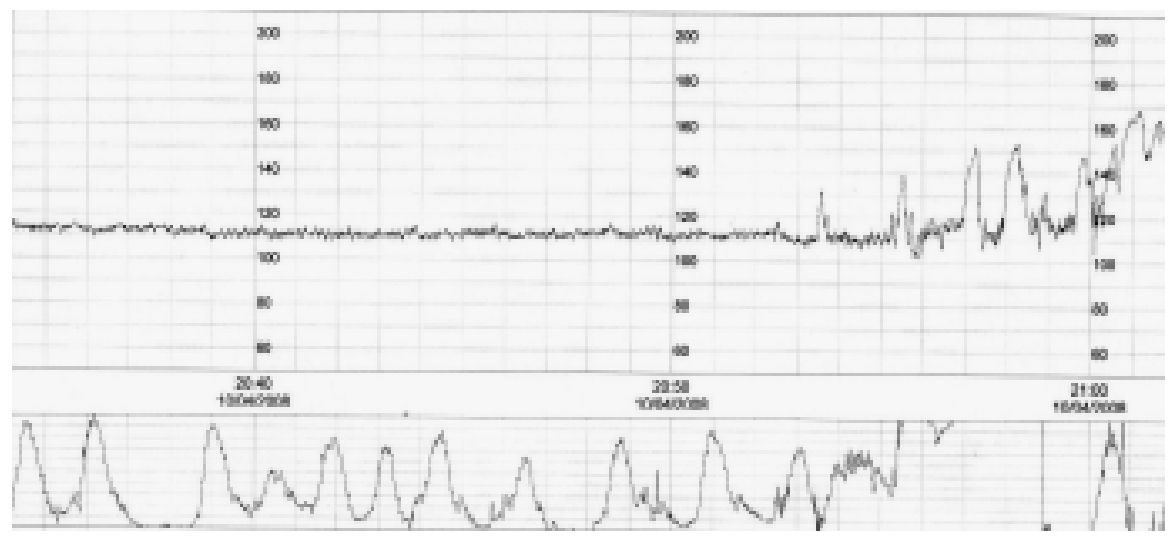

Figure 3. CTG showing 'cycling': alternating periods of quiescence and activity.

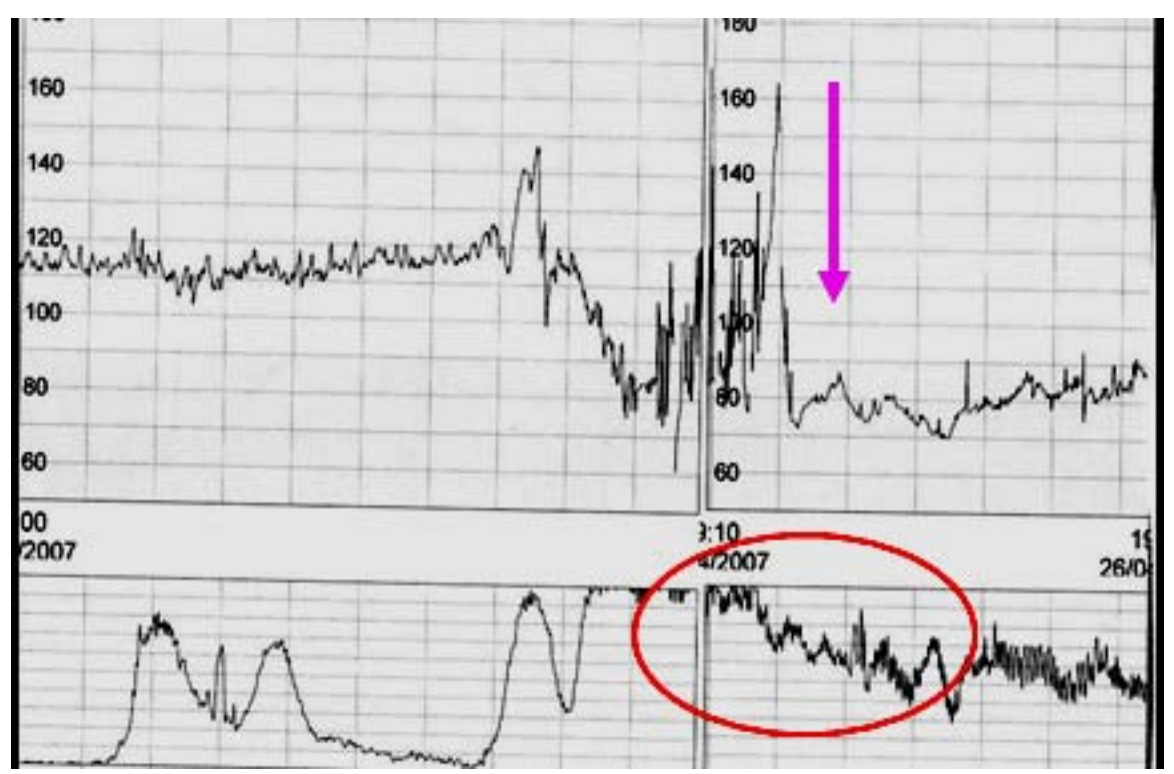

Figure 4. Acute hypoxia. 


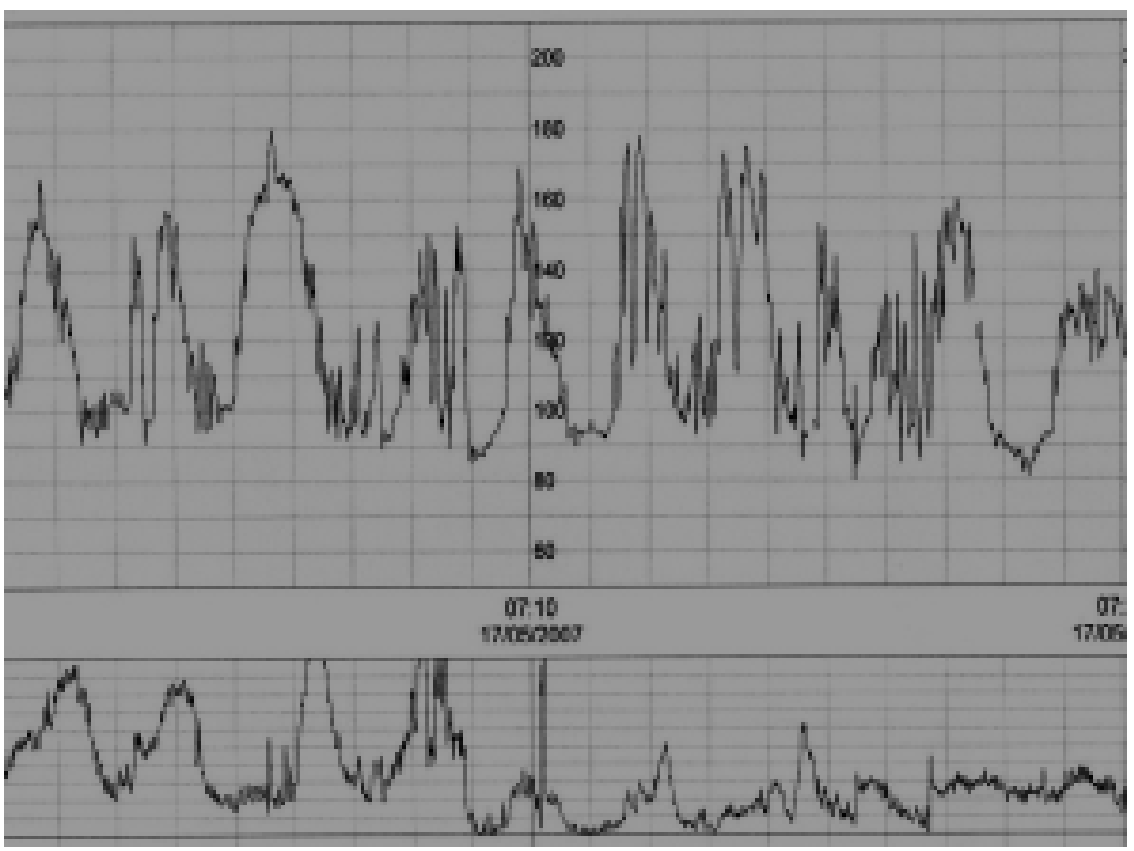

Figure 5. Sub-acute hypoxia.

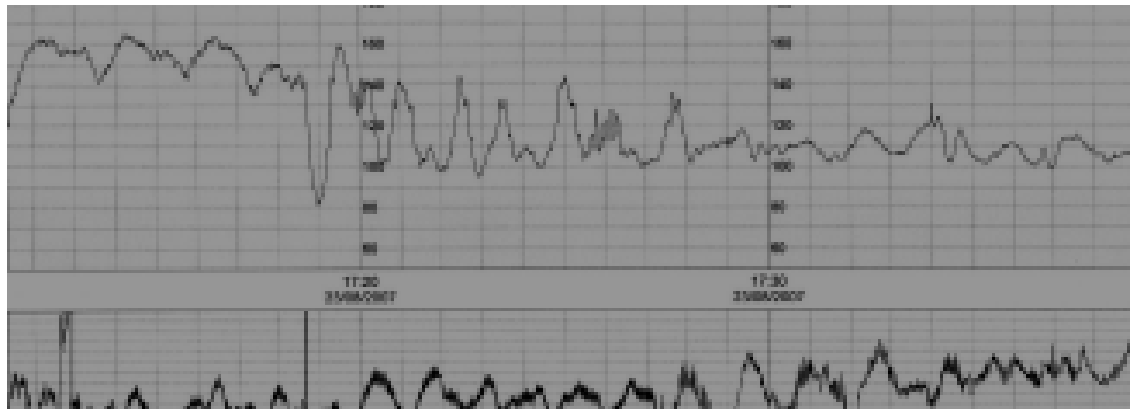

Figure 6. Gradually evolving hypoxia with 'step ladder pattern' to death.

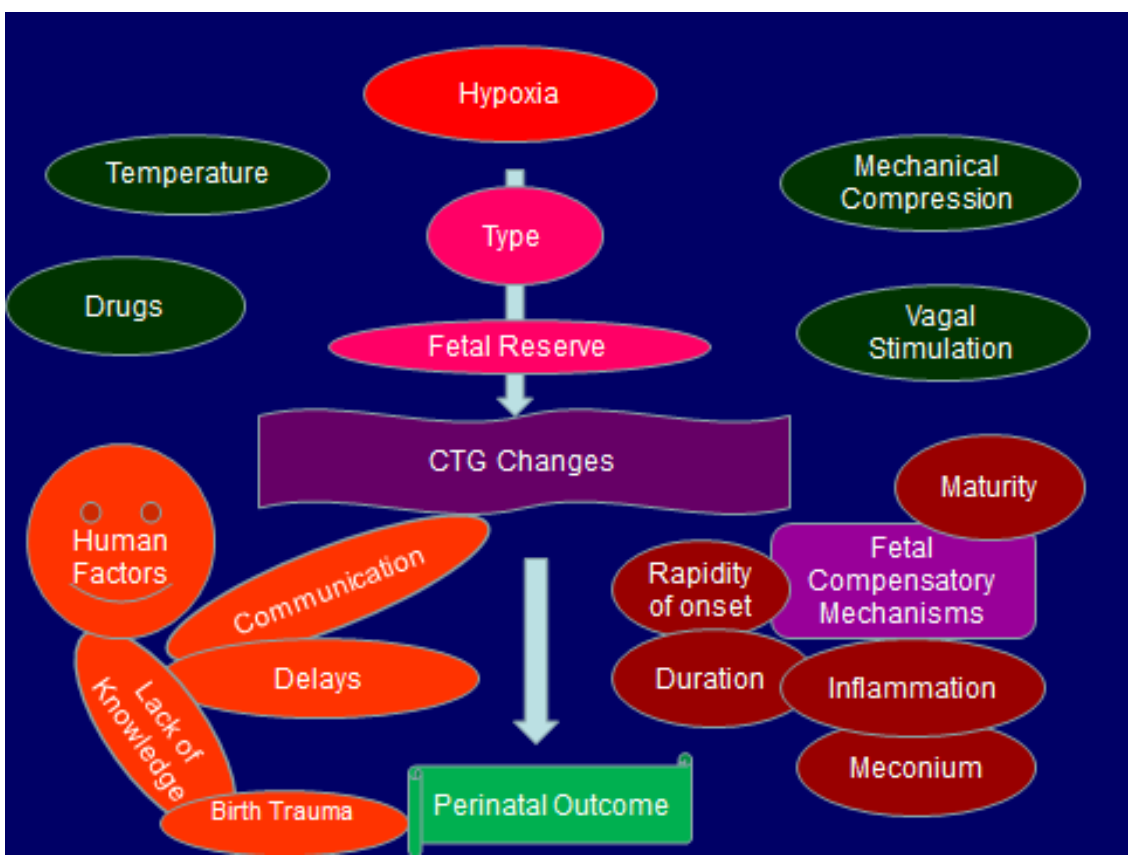

Figure 7. The 'CTG complexogram'. 


\section{Acute hypoxia}

This refers to a sudden drop in baseline heart rate and is termed 'single prolonged deceleration'. If it lasts for less than 3 minutes and then recovers to normal baseline, it is considered a suspicious feature and if it last for more than 3 minutes, it is considered to be an abnormal feature. If the heart rate remains below 80 beats/minute for over 10 minutes, then it is termed prolonged baseline bradycardia. Occurrence of a prolonged deceleration should alert the clinician to exclude three major 'accidents' during labour (abruption, cord prolapse and caesarean scar rupture) and one iatrogenic cause (uterine hyperstimulation due to oxytocin infusion). If there is any clinical evidence of these 'accidents' an immediate delivery should be undertaken to salvage the fetus. This is because metabolic acidosis is likely to get worse with time due to continued reduction in the utero-placental circulation. In the presence of acute hypoxia, the fetal $\mathrm{pH}$ has been shown to drop at the rate of $0.01 /$ minute. In cases of hyperstimulation, oxytocin infusion should be stopped immediately and tocolytics (terbulatine 250 mcg subcutaneously) administered, if required to abolish the uterine contraction ${ }^{13}$. If these three 'accidents' are excluded, it is reasonable to wait, if the variability of the heart rate during the episode of deceleration or bradycardia is normal and if the CTG prior to the deceleration was normal. Signs of recovery to the normal baseline (i.e. an 'upward trend' of the end of the deceleration, repeated attempts to reach the baseline) are also a positive feature. It is estimated that in the absence of the three 'accidents' of labour described above, over $90 \%$ of CTGs with prolonged bradycardia are likely to recover to normal baseline in 6 minutes and up to $95 \%$ in 9 minutes $^{3}$. The $3,6,9,12$, and $15^{\prime}$ minute 'guidance' on the management of prolonged decelerations is based on this observation. This guidance involves instituting appropriate interventions (positioning, hydration, tocolysis, stopping syntocinon infusion) by 6 minutes and to move the patient to theatre by 9 minutes, if the CTG shows no recovery. It is recommended that attempts at delivery should commence by 12 minutes with the aim of delivering the fetus by 15 minutes. Reduced variability prior to the onset of bradycardia has been reported to be associated with a poor outcome ${ }^{14}$. Figure 4 illustrates prolonged deceleration secondary to uterine hyperstimulation (circled) with the commencement of oxytocin. Terbutaline (arrow) was administered and the woman had a spontaneous vaginal birth 3 hours later as the fetal heart rate returned back to the normal baseline after administration of terbutaline, which abolished excessive uterine contractions.

\section{Sub-acute hypoxia}

In this situation, the fetus spends more time decelerating and progressively less time at the normal baseline fetal heart rate. Typically, the fetus spends less than 30 seconds at the baseline to 'wash off' carbon dioxide and acid and spends over 90 seconds building up carbon dioxide and acid. Figure 5 illustrates subacute hypoxia and such a fetus decompensate within minutes as the $\mathrm{pH}$ of the fetus has been shown to drop at the rate of 0.01 every 2-3 minutes.

\section{Gradually evolving hypoxia}

Hypoxic stress may develop over hours than minutes during labour and this may provide the fetus with the opportunity to utilize its compensatory mechanisms to avoid hypoxic injury. In this scenario, CTG would initially show decelerations followed by disappearance of accelerations as the fetus attempts to conserve energy by limiting muscle activity that may increase oxygen requirement. If the hypoxic insult continues, fetus then releases catecholamines to increase the heart rate and its cardiac output to supply vital organs. Despite of fetal efforts at compensation, if the hypoxic insult persists, then decompensation ensues resulting in reduced perfusion of brain leading to loss of baseline variability. The CTG would now be termed 'pre-terminal' as the final event is reduction of myocardial oxygenation that results in gradual reduction of fetal heart rate. This is often described as 'step ladder pattern to death' and signifies myocardial acidosis and failure of the autonomic centres of the brain to maintain a stable baseline heart rate, as shown in Figure 6.

\section{Long standing hypoxia and pre-terminal traces}

In both these situation, the fetus has exhausted all its reserves or is unable to compensate (e.g. intrauterine growth restriction). In the former, the hypoxic insult has occurred at some point during the antenatal period (i.e. prior to the onset of labour) and the CTG often shows a higher baseline with reduced variability and shallow decelerations with uterine contractions. Such uterine contractions during labour may cause further episodes of hypoxia and hence may worsen the existing cerebral damage. Prolonged bradycardia as well as total loss of variability (often with shallow decelerations) is often termed 'pre-terminal CTG' and these fetuses require immediate delivery.

\section{Understanding the 'CTG complexogram'}

It is essential to understand that apart from intrapartum hypoxia, many other factors such as mechanical stimulation, maternal temperature and drugs affect the fetal heart rate and hence, show CTG changes. The type of hypoxia (acute, sub-acute and gradually evolving) as well as the fetal reserve also determines the features observed on the CTG. Moreover, the observed CTG changes do not directly correlate to the ultimate perinatal outcome. Human factors (lack of knowledge, failure to incorporate 
clinical picture, delay in action and issues with communication/team working) and the capacity of the fetus to respond to hypoxic insults also contribute to perinatal outcome. Figure 7 illustrates a 'CTG complexogram' that may help clinicians to understand the variables and complexities that may affect the ultimate perinatal outcome. Clinicians often react to patterns ('early, 'late', 'variable' decelerations) observed on the CTG without understanding the type of hypoxia or the fetal response to the hypoxic stress during labour. Such an approach is likely to increase operative interventions without any changes in perinatal outcome on one hand or poor perinatal outcome due to a failure to appreciate the entire clinical picture. It is essential to understand the fetal response to hypoxic stress, especially the rise in baseline heart rate secondary to the release of catecholamines prior to planning interventions. Hence, even in centres where additional tests of fetal wellbeing such as fetal blood sampling (FBS) is not available, unnecessary operative interventions could be avoided by understanding the pathophysiology of fetal heart rate changes and the types of hypoxia during labour. This may also help medico-legal issues that may arise as a result of misinterpretation of CTG or inappropriate management ${ }^{15}$.

\section{Conclusion}

Aim of intrapartum electronic fetal monitoring using the CTG is to improve perinatal outcome without causing undue harm to the mother. Unfortunately, the use of CTG without understanding the pathophysiology of fetal hypoxia during labour and the multiple factors that may affect features observed on the CTG appears to have increased operative interventions without improving cerebral palsy or perinatal mortality rates. All clinicians who care for women during labour have a responsibility to understand the strengths and the limits of electronic fetal heart rate monitoring using CTG. Understanding the types of hypoxia, fetal reserves and other intrapartum risk factors coupled with appreciating the human factors that affect CTG interpretation may help improve perinatal outcomes and reduce unnecessary interventions, even in centres, where additional tests of fetal wellbeing are not available due to resource constraints.

\section{References}

1. Chandraharan E, Arulkumaran S. Cerebral Palsy: Aetiopathogenesis and Diagnosis. Chapter. In: Recent Advances in Obstetrics and Gynaecology - 6. Das Gupta S (ed). Jaypee Brothers 2003; pp 144-56.
2. CESDI (1997) 4th Annual Report. London. Maternal and ChildHealth Research Consortium.

3. Chandraharan E, Arulkumaran S. Prevention of birth asphyxia: responding appropriately to cardiotocograph (CTG) traces. Best Pract Res Clin Obstet Gynaecol 2007 Aug; 21(4): 609-24.

4. Chandraharan E, Arulkumaran S. Electronic fetal heart rate monitoring in current and future practice. Review Article. J ObstetGynecol India 2008; 58(2): 121-30.

5. National Institute of Clinical Excellence. Intrapartum care: care of healthy women and their babies during labour. NICE Clinical Guideline No. 55. September 2007.

6. Beard RW, Filshie GM, Knight CA, Roberts GM. The significance of the changes in the continuous fetal heart rate in the first stage of labour. J Obstet Gynecol Br C'wlth 1971; 78: $865-81$.

7. Nelson KB, Dambrosia JM, Ting TY, Grether JK. Uncertain value of electronic fetal heart rate monitoring in predicting cerebral palsy. N Engl J Med 1996; 334(10): 613-8.

8. Curzan P, Bekir JS, McLintock DG, Patel M. Reliability of cardiotocography in predicting baby's condition at birth. $\mathrm{Br}$ Med J (Clin Res Ed) 1984; 289(6455): 1345-7.

9. Chandraharan E, Arulkumaran S. Intrapartum assessment of fetal health. Chapter. In: Current Obstetrics and Gynaecology. Mukhergee GM (ed). Jaypee Brothers, 2007.

10. Westgate J, Harris M, Curnow JSH, Greene KR. Plymouth randomised trial of cardiotocogram only versus ST waveform plus cardiotocogram for intrapartum monitoring in 2400 cases. Am J ObstetGynecol 1993; 169: 1151-60.

11. Amer-Wåhlin I, Hellsten C, Norén H, Hagberg H, Herbst A, Kjellmer I, Lilja H, Lindoff C, Månsson M, Mårtensson L, Olofsson P, Sundström AK, Maršál K. Cardiotocography only versus cardiotocography plus ST analysis of fetal electrocardiogram for intrapartum fetal monitoring: a Swedish randomised controlled trial. Lancet 2001; 358: 534-38.

12. Alfirevic Z, Devane D, Gyte GML. Continuous cardiotocography (CTG) as a form of electronic fetal monitoring (EFM) for fetal assessment during labour. Cochrane Database of Systematic Reviews 2006, Issue 3. Art. No: CD006066. DOI: 10.1002/14651858.CD006066.

13. Chandraharan E, Arulkumaran S. Acute tocolysis. Review Article. Curr Opin Obstet Gynecol 2005; 17: 151-56.

14. Williams KP, Galerneau F. Fetal heart rate parameters predictive of neonatal outcome in the presence of prolonged decelerations. Obstet Gynecol 2002; 100: 951-4.

15. Chandraharan E, Arulkumaran S. Medico-legal problems in obstetrics. Current Obstetrics and Gynaecology 2006; 16: 206-10. 